\title{
Comparison of metastatic neuroendocrine neoplasms to the breast and primary invasive mammary carcinomas with neuroendocrine differentiation
}

\author{
Sambit K Mohanty ${ }^{1}$, Stacey A Kim ${ }^{1}$, Deborah F DeLair ${ }^{2}$, Shikha Bose ${ }^{1}$, Anna R Laury ${ }^{1}$, \\ Shefali Chopra ${ }^{1}$, Richard B Mertens ${ }^{1}$ and Deepti Dhall ${ }^{1}$ \\ ${ }^{1}$ Department of Pathology and Laboratory Medicine, Cedars-Sinai Medical Center, Los Angeles, CA, USA and \\ ${ }^{2}$ Department of Pathology and Laboratory Medicine, Memorial Sloan-Kettering Cancer Center, New York, \\ $N Y, U S A$
}

\begin{abstract}
Metastatic neuroendocrine neoplasms to the breast may show considerable morphologic overlap with primary mammary carcinomas, particularly those showing evidence of neuroendocrine differentiation, and may be misdiagnosed as such. Accurate distinction between these two entities is crucial for determination of appropriate clinical management. The histologic and immunohistochemical features of metastatic neuroendocrine neoplasms to the breast were studied and compared with the features of primary invasive mammary carcinomas with neuroendocrine differentiation, which served as controls. Of the metastatic neuroendocrine neoplasms, 15 were well-differentiated neuroendocrine tumors with carcinoid tumor-type morphology and 7 were poorly differentiated/high-grade neuroendocrine carcinomas with small-cell or large-cell neuroendocrine carcinoma morphology. The majority of the metastatic neoplasms originated in the lung and gastrointestinal tract. There were histologic similarities between metastatic neuroendocrine neoplasms and invasive mammary carcinomas with neuroendocrine differentiation, both of which exhibited neuroendocrine histologic features (nested and trabecular architecture, minimal tubular differentiation, and characteristic nuclear features). Only one case of the invasive mammary carcinomas with neuroendocrine differentiation was modified Bloom-Richardson grade 1 (largely due to minimal tubular differentiation on most such tumors), and the invasive mammary carcinomas with neuroendocrine differentiation were often associated with in situ carcinoma. Immunohistochemistry was helpful in distinguishing metastatic neuroendocrine neoplasms from invasive mammary carcinomas with neuroendocrine differentiation. Whereas the majority of invasive mammary carcinomas with neuroendocrine differentiation were positive for estrogen receptor and GATA3, metastatic neuroendocrine neoplasms were typically negative for estrogen receptor and GATA3, and metastatic well-differentiated neuroendocrine tumors often showed immunoreactivity for site-specific markers. Although the histologic and immunohistochemical features of a breast tumor may raise the suspicion of a metastatic neuroendocrine neoplasm, the pathologic findings should be interpreted in the context of the clinical history and imaging findings in order to establish an accurate diagnosis. Modern Pathology (2016) 29, 788-798; doi:10.1038/modpathol.2016.69; published online 29 April 2016
\end{abstract}

Metastases to the breast are unusual, comprising less than $1 \%$ of all malignant neoplasms of the breast. $^{1-5}$ The majority of the metastatic lesions originate from the contralateral breast or hematopoietic malignancies. ${ }^{1,2,5}$ Stomach, kidney, ovary,

Correspondence: Dr D Dhall, MD, Department of Pathology and Laboratory Medicine, Cedars-Sinai Medical Center, 8700 Beverly Blvd, Los Angeles, CA 90048, USA.

E-mail: Deepti.Dhall@cshs.org

Received 18 September 2015; revised 24 February 2016; accepted 6 March 2016; published online 29 April 2016 lung, and skin are the other common primary sites. ${ }^{1-5}$ Metastatic neuroendocrine neoplasms to the breast are rare and comprise only $1-2 \%$ of all metastatic tumors to the breast. To the best of our knowledge, less than 50 cases have been described to date, primarily in the form of isolated case reports and rare case series. ${ }^{4-24}$

Metastatic neuroendocrine neoplasms to the breast may show considerable morphologic overlap with in situ and invasive mammary carcinomas, particularly those showing evidence of neuroendocrine differentiation. Accurate distinction between these 
two entities has important management implications and is crucial in order to avoid unnecessary procedures and treatments. In the absence of a known clinical history of a primary extramammary neuroendocrine neoplasm, a metastatic neuroendocrine neoplasm in the breast may potentially be misdiagnosed as an invasive mammary carcinoma in routine surgical pathology practice, and the patient may be subjected to unnecessary mastectomy and/or axillary node biopsy or dissection, with or without radiation/chemotherapy, based on the mistaken diagnosis of primary mammary carcinoma.

This study was undertaken to characterize the clinicopathologic features of metastatic neuroendocrine neoplasms to the breast and compare them with the features of primary invasive mammary carcinomas with neuroendocrine differentiation. In addition, we sought to determine the utility of immunohistochemistry in distinguishing these two entities.

\section{Materials and methods}

After approval from the Institutional Review Board, the anatomic pathology database of Cedars-Sinai Medical Center (Los Angeles, CA, USA) was searched from January 1990 to June 2013 for metastatic neuroendocrine neoplasms (including well-differentiated neuroendocrine neoplasms, also known as well-differentiated neuroendocrine tumors, and poorly differentiated neuroendocrine neoplasms, also referred to as high-grade neuroendocrine carcinomas) to the breast. Since the main focus of this study was to identify features that can differentiate metastatic neuroendocrine neoplasms in the breast from primary breast tumors with similar features, we searched the pathology archives from the same time interval for primary invasive breast tumors that were reported to exhibit neuroendocrine differentiation. We further limited our selection of a control group to include only those cases that satisfied the 2003 WHO definition of invasive mammary carcinoma with neuroendocrine differentiation ${ }^{25}$ (presence of morphologic evidence of neuroendocrine differentiation and greater than $50 \%$ positivity for at least one neuroendocrine marker). Since the selected cases of invasive mammary carcinomas with neuroendocrine differentiation served as a control group, we studied only 22 consecutive cases, in order to match the number of metastatic neuroendocrine neoplasms. For metastatic neuroendocrine neoplasms, both in-house cases and consultation cases referred from outside institutions were included in this study. Since metastatic neuroendocrine neoplasms to the breast are very rare, we also included eight additional recently published cases of metastatic neuroendocrine neoplasms to the breast from the anatomic pathology database of Memorial Sloan-Kettering Cancer Center (New York, NY, USA). ${ }^{4}$

The clinical histories, including results of imaging studies and prior pathology reports, were reviewed in detail to ascertain the site of origin of metastatic neuroendocrine neoplasms to the breast. Cases for which a definite primary site of origin could not be determined with certainty were excluded from the study.

The hematoxylin and eosin (H\&E)-stained slides and immunohistochemical stains from each case were reviewed by two pathologists (SKM and SAK) to confirm the diagnosis. Histopathologic data including the grade of the metastatic neuroendocrine neoplasms, the histologic type and modified BloomRichardson histopathologic grade of the primary mammary tumors, and the presence of in situ carcinoma were recorded. Histologic grading of the metastatic neuroendocrine neoplasms was performed on the basis of mitotic count and Ki-67 proliferative index. ${ }^{26-28}$ Subsequently, the slides were reviewed in a blinded fashion by a pathologist with expertise in neuroendocrine tumors (DD), who tabulated the histologic features (including architectural patterns and detailed nuclear and cytoplasmic features) for each case. The results of previously performed immunohistochemical stains for estrogen receptor (ER), progesterone receptor (PR), Ki-67, chromogranin, synaptophysin, and Her-2/neu and previously performed Her-2/neu amplification studies by fluorescence in situ hybridization (FISH) were reviewed and recorded, as were the results of immunohistochemical stains for site-specific markers such as thyroid transcription factor-1 (TTF-1) and CDX2, which had been performed on cases of metastatic neuroendocrine neoplasm to the breast. In addition, GATA3 immunostain was performed in 9 cases of metastatic neuroendocrine neoplasm to the breast and 18 cases of invasive mammary carcinoma with neuroendocrine differentiation, and TTF-1 and CDX2 immunostains were performed on the same 18 cases of invasive mammary carcinoma with neuroendocrine differentiation in which GATA3 was performed (see Table 1 for antibody specifications).

The extent of immunoreactivity for the neuroendocrine markers was graded as follows: no cells staining = negative $; \quad<50 \%$ of cells staining = focal; $>50 \%$ of cells staining $=$ diffuse. For the hormone receptors, GATA3, TTF-1, and CDX2, nuclear staining in $\geq 1 \%$ of tumor cells was considered as positive. The intensity of immunoreactivity for all the stains was graded as follows: weak, moderate, and strong. The Ki-67 proliferative index was assessed using a computer-assisted image analysis system in most cases from our institution. ${ }^{29,30}$

\section{Results}

\section{Clinical Characteristics of Metastatic Neuroendocrine Neoplasms to the Breast}

Twenty-two cases of metastatic neuroendocrine neoplasm to the breast were identified, including 14 from Cedars-Sinai Medical Center and 8 from 
Table 1 Immunohistochemical markers, clones, and protocols

\begin{tabular}{|c|c|c|c|c|c|c|}
\hline Antibodies & Vendor & Clone & Dilution & Pretreatment & Detection & Instrument \\
\hline GATA3 & $\begin{array}{l}\text { Biocare (Concord, } \\
\text { CA, USA) }\end{array}$ & $\begin{array}{l}\text { Clone L50-823 } \\
\text { (mouse monoclonal) }\end{array}$ & 1:600 dilution & $\begin{array}{l}\text { Onboard heat-induced } \\
\text { epitope retrieval high } \\
\text { pH CC1 buffer }\end{array}$ & $\begin{array}{l}\text { Ventana } \\
\text { Ultraview DAB }\end{array}$ & $\begin{array}{l}\text { Ventana Benchmark } \\
\text { Ultra (Tucson, AZ, USA) }\end{array}$ \\
\hline TTF-1 & $\begin{array}{l}\text { Ventana (Tucson, } \\
\text { AZ, USA) }\end{array}$ & $\begin{array}{l}\text { SP141 (rabbit } \\
\text { monoclonal) }\end{array}$ & Prediluted & $\begin{array}{l}\text { Onboard heat-induced } \\
\text { epitope retrieval high } \\
\text { pH CC1 buffer }\end{array}$ & $\begin{array}{l}\text { Ventana } \\
\text { Ultraview DAB }\end{array}$ & $\begin{array}{l}\text { Ventana Benchmark } \\
\text { Ultra (Tucson, AZ, USA) }\end{array}$ \\
\hline CDX2 & $\begin{array}{l}\text { Cell Marque } \\
\text { (Rocklin, CA, USA) }\end{array}$ & $\begin{array}{l}\text { EPR2764Y (rabbit } \\
\text { monoclonal) }\end{array}$ & Prediluted & $\begin{array}{l}\text { Onboard heat-induced } \\
\text { epitope retrieval high } \\
\text { pH CC1 buffer }\end{array}$ & $\begin{array}{l}\text { Ventana } \\
\text { Ultraview DAB }\end{array}$ & $\begin{array}{l}\text { Ventana Benchmark } \\
\text { Ultra (Tucson, AZ, USA) }\end{array}$ \\
\hline
\end{tabular}

Memorial Sloan-Kettering Cancer Center. The clinical characteristics of these tumors are summarized in Table 2.

Fifteen patients (68\%) had a known prior history of neuroendocrine neoplasm when they presented with breast lesion(s), which were correctly diagnosed as metastatic neuroendocrine neoplasms. In two patients in whom the initial clinical presentation was a breast mass, the correct diagnosis of metastatic low-grade neuroendocrine tumor was suggested based on the morphologic and immunohistochemical features of the tumors. A subsequent search for a primary tumor revealed the site of origin to be the terminal ileum in both patients. In two patients initially presenting with both a breast mass and a lung mass, a correct diagnosis of primary lung neuroendocrine neoplasm (one a small-cell carcinoma and the other an atypical carcinoid) metastatic to breast was rendered based on the clinical, histologic, and immunohistochemical findings.

Three of 22 patients with metastatic neuroendocrine neoplasms to the breast (14\%) were initially misdiagnosed as having a primary invasive mammary carcinoma. One patient was initially misdiagnosed with a low-grade primary invasive mammary carcinoma and treated with chemotherapy and radiation therapy. A year later, while she was being worked up for pneumonia, multiple liver lesions were discovered which were proven on biopsy to be metastatic low-grade neuroendocrine tumor. Further evaluation identified the small bowel as the primary site of origin. Her initial breast tumor was then retrospectively reviewed and reclassified as metastatic neuroendocrine tumor. The second patient presented with a breast mass as well as multiple liver lesions. The breast mass was initially misdiagnosed as invasive ductal carcinoma. However, a concurrent liver biopsy revealed metastatic neuroendocrine tumor of presumed small bowel origin, and the diagnosis of the breast tumor was amended after rereview demonstrated neuroendocrine differentiation in the breast tumor. The third patient initially presented with a breast mass, which was diagnosed as a primary mammary carcinoma. It was reclassified as metastatic neuroendocrine neoplasm after the discovery of metastatic neuroendocrine neoplasm in the liver from a small bowel primary.

\section{Clinical Characteristics of Primary Invasive Mammary Carcinomas with Neuroendocrine Differentiation}

The clinical characteristics of the 22 cases of primary invasive mammary carcinoma with neuroendocrine differentiation are summarized in Table 2. The mean age of patients with invasive mammary carcinoma with neuroendocrine differentiation (62 years) was similar to the mean age of the patients with metastatic neuroendocrine neoplasm (60 years).

\section{Pathologic Characteristics of Metastatic Neuroendocrine Neoplasms to the Breast}

The pathologic features of the 22 cases of metastatic neuroendocrine neoplasm to the breast are summarized in Tables 3 and 4 . The tumors ranged from 0.4 to $14.0 \mathrm{~cm}$ in greatest dimension. On microscopic evaluation, there were 15 well-differentiated neuroendocrine tumors and 7 poorly differentiated/ high-grade neuroendocrine carcinomas. Among gastrointestinal well-differentiated neuroendocrine tumors, there were two low-grade and six intermediate-grade tumors. Among well-differentiated neuroendocrine tumors of lung origin, there were two typical carcinoids and four atypical carcinoids. Except for two cases (one from the colon and one from the ileum), all well-differentiated neuroendocrine tumors were from our institution, and except for one small-cell carcinoma from the lung, all high-grade neuroendocrine carcinomas were from Memorial Sloan-Kettering Cancer Center.

The tumor cells of all well-differentiated neuroendocrine tumors were arranged predominantly in the form of nests, trabeculae, cords, and/or pseudoacini. Individual neoplastic cells were uniform with round to oval, smooth-contoured nuclei, inconspicuous nucleoli, characteristic 'salt and pepper'-like nuclear chromatin, and a moderate amount cytoplasm. Cytoplasmic granularity was variable; it was most prominent in tumors of small bowel origin. Focal single cell/punctuate necrosis was 
Table 2 Clinical characteristics of patients with metastatic neuroendocrine neoplasms to the breast and invasive mammary carcinomas with neuroendocrine differentiation

\begin{tabular}{|c|c|c|}
\hline Characteristics & $\begin{array}{l}\text { Metastatic neuroendocrine neoplasms to the breast } \\
(\mathrm{n}=22)\end{array}$ & $\begin{array}{l}\text { Invasive mammary carcinomas with neuroendocrine } \\
\text { differentiation }(\mathrm{n}=22)\end{array}$ \\
\hline Mean age (range) in years & $60(28-82)$ & $62(40-87)$ \\
\hline Sex & 21 females; 1 male & 22 females \\
\hline Site of origin & $\begin{array}{l}\text { Lung (11); gastrointestinal tract (8); cervix (1); } \\
\text { endometrium (1); ovary (1) }\end{array}$ & Breast (22) \\
\hline Tumor laterality & Unilateral (17): right $=8$, left $=9$; bilateral $(5)$ & Unilateral (21): right =11, left =10; bilateral (1) \\
\hline Specimen type & $\begin{array}{l}\text { Needle core biopsy (11); lumpectomy (8); } \\
\text { mastectomy (2); autopsy (1) }\end{array}$ & $\begin{array}{l}\text { Needle core biopsy (3); lumpectomy (12); mastectomy } \\
\text { (7) }\end{array}$ \\
\hline
\end{tabular}

Table 3 Macroscopic and histopathologic characteristics of metastatic neuroendocrine neoplasms to the breast and invasive mammary carcinomas with neuroendocrine differentiation

\begin{tabular}{|c|c|c|}
\hline Characteristics & $\begin{array}{l}\text { Metastatic neuroendocrine neoplasms } \\
\text { to the breast }(\mathrm{n}=22)\end{array}$ & $\begin{array}{l}\text { Invasive mammary carcinomas with } \\
\text { neuroendocrine differentiation }(\mathrm{n}=22)\end{array}$ \\
\hline Tumor size (range, mean, and median) & $0.4-14 \mathrm{~cm}(\text { mean }=2.4 \mathrm{~cm} ; \text { median }=0.9 \mathrm{~cm})^{\mathrm{a}}$ & $0.5-7.5 \mathrm{~cm}($ mean $=2.4 \mathrm{~cm} ;$ median $=2.1 \mathrm{~cm})$ \\
\hline \multirow{2}{*}{ Tumor type } & Well-differentiated $(n=15)$ & Ductal $(n=21)$ \\
\hline & Poorly-differentiated $(n=7)$ & Lobular $(n=1)$ \\
\hline \multirow[t]{3}{*}{ Tumor grade ${ }^{\mathrm{b}}$} & Low-grade/typical carcinoid $(n=4)$ & MBR low-grade $(n=1)$ \\
\hline & Intermediate-grade/atypical carcinoid $(n=10)$ & MBR intermediate-grade $(n=13)$ \\
\hline & High-grade $(n=7)$ & MBR high-grade $(n=8)$ \\
\hline In situ component & None & $\begin{array}{l}\text { Ductal carcinoma in situ }(n=15) \\
\text { Lobular carcinoma in situ }(n=0)\end{array}$ \\
\hline
\end{tabular}

Abbreviation: MBR, modified Bloom-Richardson.

${ }^{\text {a }}$ The tumor size was available in nine cases.

${ }^{\mathrm{b}}$ One case of metastatic neuroendocrine (carcinoid) tumor from ovary is not graded due to the lack of grading criteria.

identified in two atypical carcinoids. Mitotic figures were few in low-grade/typical carcinoid tumors (by definition less than 2 mitoses/10 high-power fields) and ranged from 2 to 10 mitoses/10 high-power fields in intermediate-grade/atypical carcinoid tumors.

The tumor cells of all seven high-grade neuroendocrine carcinomas were arranged in solid nests and/or confluent sheets. The neoplastic cells were either small to intermediate in size with increased nuclear-to-cytoplasmic ratio and scanty cytoplasm or large polygonal cells with vesicular nuclei, prominent nucleoli and chromocenters, and a moderate amount of cytoplasm. Small-cell neuroendocrine features were observed in four tumors, and largecell neuroendocrine features were seen in three cases. Areas of necrosis were also identified in all high-grade neuroendocrine carcinomas, ranging from focal in three cases to widespread and/or geographic in four cases. Nuclear molding and crush artifact/nuclear smearing were appreciated in tumors with small-cell histology. All high-grade neuroendocrine carcinomas showed numerous mitotic figures (greater than 20 mitoses/10 high-power fields).

None of the metastatic neuroendocrine neoplasms was associated with either ductal or lobular carcinoma in situ.

\section{Pathologic Characteristics of Primary Invasive Mammary Carcinomas with Neuroendocrine Differentiation}

The pathologic features of the 22 cases of invasive mammary carcinomas with neuroendocrine differentiation are summarized in Table 3 . The tumors ranged from 0.5 to $7.5 \mathrm{~cm}$ in greatest dimension. On microscopic evaluation, neuroendocrine features were seen in all tumors, including arrangement of tumor cells in solid nests, trabeculae, cords and/or acinar structures. The nuclei were round to oval, with variable degrees of atypia, having smooth to irregular nuclear membranes. In most cases, 'salt and pepper'-like granular nuclear chromatin was appreciated, at least focally. In others, prominent nucleoli were noted. Most tumors displayed a moderate amount of cytoplasm with faint granularity, though rarely the cytoplasmic granularity was more prominent. The cytoplasm was scanty in rare cases. Only one case exhibited focal mucinous differentiation. Except for one tumor with features of invasive lobular carcinoma, all were classified as ductal type. None of the invasive mammary carcinomas with neuroendocrine differentiation displayed typical features of small-cell carcinoma or large-cell neuroendocrine carcinoma. Using the modified Bloom-Richardson grading system, 8 of the 22 invasive mammary carcinomas with neuroendocrine 


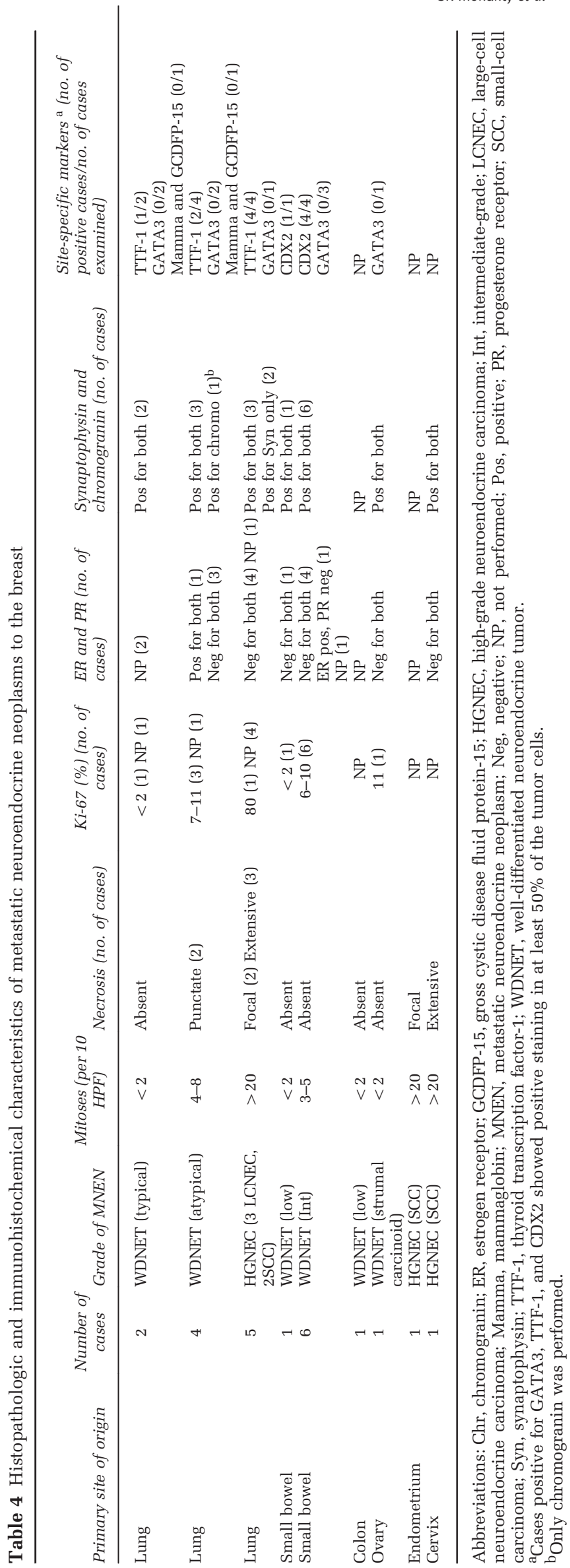

differentiation were classified as high-grade tumors, 13 as intermediate-grade tumors, and 1 as a lowgrade tumor. The tubule score was 3 in all tumors except for one case in which the tubule score was 2 . The nuclear score was 2 in 19 tumors and 3 in 3 tumors. Mitotic activity ranged from 4 mitoses to 41 mitoses/10 high-power fields (mitotic score $=1$ in 12 cases, 2 in 2 cases, and 3 in 8 cases). Ductal carcinoma in situ was present in 15 cases (68\%), including 1 case of low-grade ductal carcinoma in situ, 10 cases of intermediate-grade ductal carcinoma in situ, and 4 cases of high-grade ductal carcinoma in situ. No lobular carcinoma in situ was seen in any of the specimens with invasive mammary carcinoma with neuroendocrine differentiation, including the case demonstrating invasive lobular carcinoma in a needle core biopsy specimen.

\section{Immunohistochemical Characteristics of Metastatic Neuroendocrine Neoplasms to the Breast}

Results of immunostains and Her-2 FISH studies performed to further characterize the metastatic neuroendocrine neoplasms are summarized in Tables 4 and 5. Nineteen cases were evaluated for synaptophysin immunoreactivity, all of which $(100 \%)$ were positive, and 20 cases were evaluated for chromogranin immunoreactivity, 18 (90\%) of which were positive. Diffuse and strong staining for both synaptophysin and chromogranin was observed in $15(79 \%)$ of the 19 cases stained for both markers (Figure 1). Immunostaining for ER and PR was performed in 16 cases. Two cases $(13 \%)$ exhibited positive staining for ER, including one tumor originating from the lung which displayed weak reactivity for ER in $40 \%$ of the tumor cells and one tumor from the gastrointestinal tract which displayed moderate reactivity for ER in 10\% of the tumor cells. One case (6\%), the aforementioned metastatic neuroendocrine neoplasm originating in the lung, exhibited weak positivity for PR in $30 \%$ of the tumor cells. Her-2/neu immunohistochemistry was performed in six cases, all of which were negative $($ score $=0)$. Her-2 FISH was done on two cases, both of which were negative for Her-2 amplification. The Ki-67 proliferative index was determined in 13 cases, the results of which are shown in Tables 4 and 5. TTF-1 immunostain was positive in 7 of $10(70 \%)$ examined metastatic neuroendocrine neoplasms of lung origin, and CDX2 immunostain was positive in all $5(100 \%)$ examined metastatic neuroendocrine neoplasms of small bowel origin. GATA3 immunostaining was performed in nine cases, all of which were negative, including the one lung tumor that was positive for ER (Figure 1). Immunostains for mammaglobin and gross cystic disease fluid protein-15 (GCDFP-15) performed in two metastatic neuroendocrine neoplasms from the lung were negative. The differences in immunohistochemical 
Table 5 Immunohistochemical and FISH characteristics of metastatic neuroendocrine neoplasms to the breast and invasive mammary carcinomas with neuroendocrine differentiation

\begin{tabular}{|c|c|c|}
\hline & $\begin{array}{l}\text { Metastatic neuroendocrine neoplasms to the } \\
\text { breast }(n=22)\end{array}$ & $\begin{array}{l}\text { Primary invasive mammary carcinomas with } \\
\text { neuroendocrine differentiation }(n=22)\end{array}$ \\
\hline Estrogen receptor positivity ${ }^{\mathrm{a}}$ & $\begin{array}{l}2 / 16(13 \%) \text {, intensity: weak to moderate, } \\
\text { extent: } 40 \text { and } 10 \%\end{array}$ & $\begin{array}{l}\text { 20/22 (91\%), intensity: moderate to strong, } \\
\text { extent: } 80-100 \%\end{array}$ \\
\hline Progesterone receptor positivity ${ }^{\mathrm{a}}$ & $1 / 16$ (6\%), intensity: weak, extent: $30 \%$ & $\begin{array}{l}15 / 22(68 \%) \text {, intensity: weak to strong, extent: } \\
20-98 \%\end{array}$ \\
\hline Her-2/neu results (IHC and/or FISH) & $\begin{array}{l}\text { 6/6 ( } 100 \%) \text { : negative (0) by IHC, } 2 / 2(100 \%) \text { : } \\
\text { not amplified by FISH }\end{array}$ & $\begin{array}{l}1 / 22(5 \%): 3+\text { by IHC, amplified by FISH; } 1 / 22 \\
(5 \%): 2+\text { by IHC, not amplified by FISH; } 20 / 22 \\
(91 \%) \text { : negative ( } 0 \text { or } 1+\text { ) by IHC, not amplified by } \\
\text { FISH }\end{array}$ \\
\hline GATA3 positivity ${ }^{\mathrm{b} / \mathrm{a}}$ & $0 / 9(0 \%)$ & $18 / 18(100 \%)$ \\
\hline Synaptophysin positivity ${ }^{\mathrm{a}}$ & $\begin{array}{l}\text { 19/19 }(100 \%) \text {, diffuse and strong staining } \\
\text { ( } n=13 \text { WDNET, } n=3 \text { HGNEC), focal and } \\
\text { strong staining ( } n=3 \text {, both HGNEC) }\end{array}$ & $\begin{array}{l}20 / 22(91 \%) \text {, diffuse and strong staining }(n=18) \text {, } \\
\text { diffuse and moderate staining }(n=1) \text {, focal and } \\
\text { strong }(n=1)\end{array}$ \\
\hline Chromogranin positivity ${ }^{\mathrm{a}}$ & $\begin{array}{l}18 / 20(90 \%), \text { diffuse and strong staining } \\
(n=14 \text { WDNET, } n=2 \text { HGNEC), focal and } \\
\text { strong staining }(n=2 \text {, both HGNEC) }\end{array}$ & $\begin{array}{l}22 / 22(100 \%) \text {, diffuse and strong staining }(n=13) \text {, } \\
\text { diffuse and moderate staining }(n=1) \text {, focal and } \\
\text { strong }(n=6) \text {, focal and weak }(n=2)\end{array}$ \\
\hline $\begin{array}{l}\text { Synaptophysin and chromogranin } \\
\text { positivity in a diffuse pattern with } \\
\text { strong intensity }\end{array}$ & $15 / 19(79 \%)$ & $10 / 22(45 \%)$ \\
\hline Ki-67 index & $\begin{array}{l}\text { Range: }<2-80 \%(n=13),<2 \%(n=2), 2- \\
20 \%(n=10), 21-50 \%(n=0),>50 \%(n=1)\end{array}$ & $\begin{array}{l}\text { Range: } 1-86 \%(n=20),<14 \%(n=9),>14 \% \\
(n=11)\end{array}$ \\
\hline
\end{tabular}

Abbreviations: FISH, fluorescence in situ hybridization; HGNEC, high-grade neuroendocrine carcinoma; IHC, immunohistochemistry; WDNET, well-differentiated neuroendocrine tumor.

${ }^{\mathrm{a}}$ No. of positive cases/no. of cases examined.

bAll GATA3 positive cases showed diffuse and strong nuclear immunoreactivity.

characteristics of the metastatic high-grade neuroendocrine carcinomas and the metastatic welldifferentiated neuroendocrine tumors are demonstrated in Table 4.

\section{Immunohistochemical Characteristics and Her-2/neu FISH Results of Primary Invasive Mammary Carcinomas with Neuroendocrine Differentiation}

Results of immunostains and Her-2 FISH studies performed on invasive mammary carcinomas with neuroendocrine differentiation are summarized in Table 5. Immunostains for ER, PR, and Her-2/neu and FISH studies for Her-2/neu amplification were performed on all 22 cases. Twenty (91\%) of the cases showed positive staining for ER and 15 (68\%) of the cases were positive for progesterone receptor (Figure 2). Except for two cases, all cases were negative for Her-2/neu by immunohistochemistry. Her-2/neu amplification by FISH was identified in one $(5 \%)$ of the cases, which showed strong $(3+)$ immunohistochemical staining for Her-2/neu. Only one case was triple-negative. Ki-67 proliferative index was determined on 20 of the 22 cases and ranged from 1 to $86 \%$. Twenty (91\%) of the 22 cases exhibited positive staining for synaptophysin and all $22(100 \%)$ cases were positive for chromogranin. Diffuse and strong immunoreactivity for both neuroendocrine markers was noted in $10(45 \%)$ of the 22 cases (Figure 2). The 18 tumors stained for GATA3, TTF-1, and CDX2 exhibited diffuse and strong nuclear immunoreactivity for GATA3 and negativity for TTF-1 and CDX2 (Figure 2).

\section{Discussion}

Metastatic neuroendocrine neoplasms to the breast are rare and may be misdiagnosed as primary invasive mammary carcinoma, resulting in inappropriate surgical intervention or other therapy. Factors that may contribute to the misdiagnosis of metastatic neuroendocrine neoplasm to the breast include the rarity of such tumors, the morphologic resemblance of these tumors to invasive as well as in situ mammary carcinomas, a lower level of awareness of their existence, and the lack of definitive diagnostic criteria for establishing the diagnosis of metastatic neuroendocrine neoplasm to the breast. This differential diagnostic problem is further compounded by the fact that primary mammary carcinomas may exhibit neuroendocrine differentiation. ${ }^{25,31-33}$

Invasive mammary carcinomas with neuroendocrine differentiation are being increasingly recognized, and differentiating them from metastatic neuroendocrine neoplasms can be difficult, particularly in the absence of a clinical history of a prior or concurrent extramammary neuroendocrine neoplasm. In this study, we compared the histopathologic and immunohistochemical features of metastatic neuroendocrine neoplasms to the breast with invasive mammary carcinomas with neuroendocrine differentiation; to our knowledge, such a study has not been previously reported. We elected to limit our study to a comparison of metastatic neuroendocrine neoplasms with invasive mammary carcinomas in which morphologic features suggestive of 

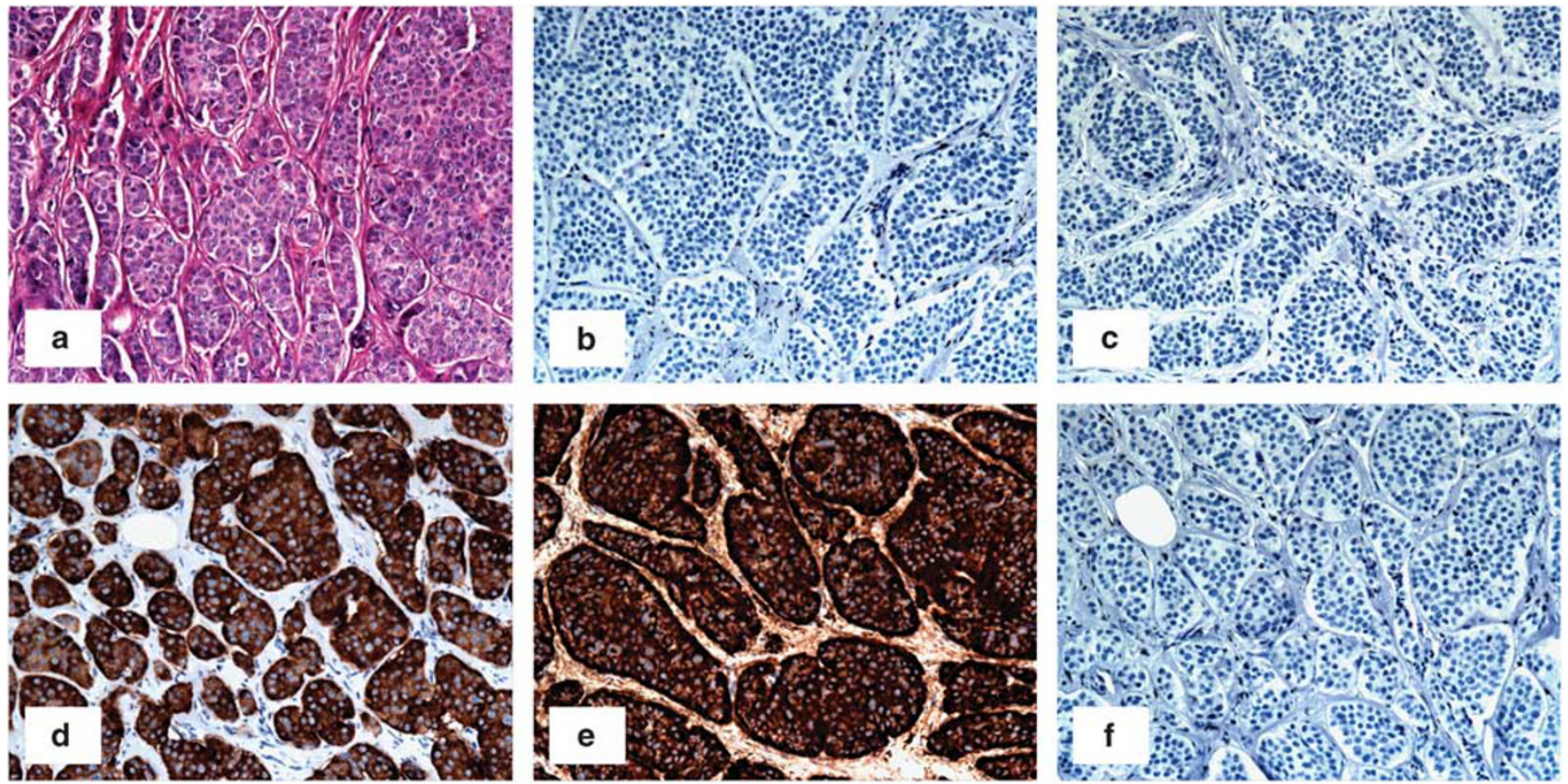

Figure 1 Metastatic well-differentiated neuroendocrine neoplasm (magnification $\times 200$ ); (a) H\&E stain showing nested and trabecular architecture; (b) negative staining for ER; (c) negative staining for PR; (d) diffuse and strong immunoreactivity for synaptophysin; (e) diffuse and strong immunoreactivity for chromogranin; and (f) negative staining for GATA3.

neuroendocrine differentiation were evident at the time of original sign-out, prompting the pathologist to evaluate the tumors for immunohistochemical evidence of neuroendocrine differentiation, and which satisfied the WHO (2003) criteria for invasive mammary carcinoma with neuroendocrine differentiation (presence of morphologic evidence of neuroendocrine differentiation and greater than $50 \%$ positivity for at least one neuroendocrine marker). ${ }^{25}$ Although the current (2012) WHO criteria $^{34}$ for diagnosis of invasive mammary carcinoma with neuroendocrine differentiation do not require a minimum percentage of cells exhibiting neuroendocrine marker positivity, we intentionally chose to retain the $50 \%$ threshold of immunohistochemical expression of immunohistochemical markers specified in the 2003 WHO criteria, in order to select as a control group the subset of invasive mammary carcinomas with neuroendocrine differentiation which most closely resembles metastatic neuroendocrine neoplasms, in terms of both morphological features and expression of markers of neuroendocrine differentiation.

We observed considerable morphologic overlap between metastatic neuroendocrine neoplasms and invasive mammary carcinomas with neuroendocrine differentiation, and $3(14 \%)$ of the 22 metastatic neuroendocrine neoplasms were initially misdiagnosed as invasive mammary carcinomas. In a previous study of metastatic neuroendocrine tumors to the breast, Perry et al. ${ }^{9}$ reported a much higher rate $(44 \%)$ of initial misdiagnosis. To avoid making this mistake, it is critical to look for neuroendocrine features when evaluating any tumor in the breast; if such features are present, invasive mammary carcinoma with neuroendocrine differentiation needs to be differentiated from metastatic neuroendocrine neoplasm. If not already provided, a pertinent clinical history must be obtained in such cases, since the pathologist's knowledge of a coexistent or prior neuroendocrine neoplasm will greatly aid in the diagnosis of metastatic neuroendocrine neoplasm to the breast.

Our study revealed some histologic features that may be helpful in differentiating between the two entities. (1) In comparison with metastatic welldifferentiated neuroendocrine tumors, invasive mammary carcinomas with neuroendocrine differentiation often display irregular nuclear membranes, a greater degree of nuclear atypia/pleomorphism, and frequent mitotic activity. These features, if present, are more in favor of invasive mammary carcinoma with neuroendocrine differentiation. On the other hand, if a tumor in the breast exhibits nested/organoid architecture with uniform nuclei, smooth nuclear membranes, minimal to no nuclear pleomorphism, and 'salt and pepper'-like nuclear chromatin throughout most of the tumor, the possibility of metastatic well-differentiated neuroendocrine tumor must be considered. In some cases, however, invasive mammary carcinomas with neuroendocrine differentiation may be histologically indistinguishable from metastatic well-differentiated neuroendocrine tumors. (2) None of the metastatic neuroendocrine neoplasms were associated with either ductal or lobular carcinoma in situ in the 

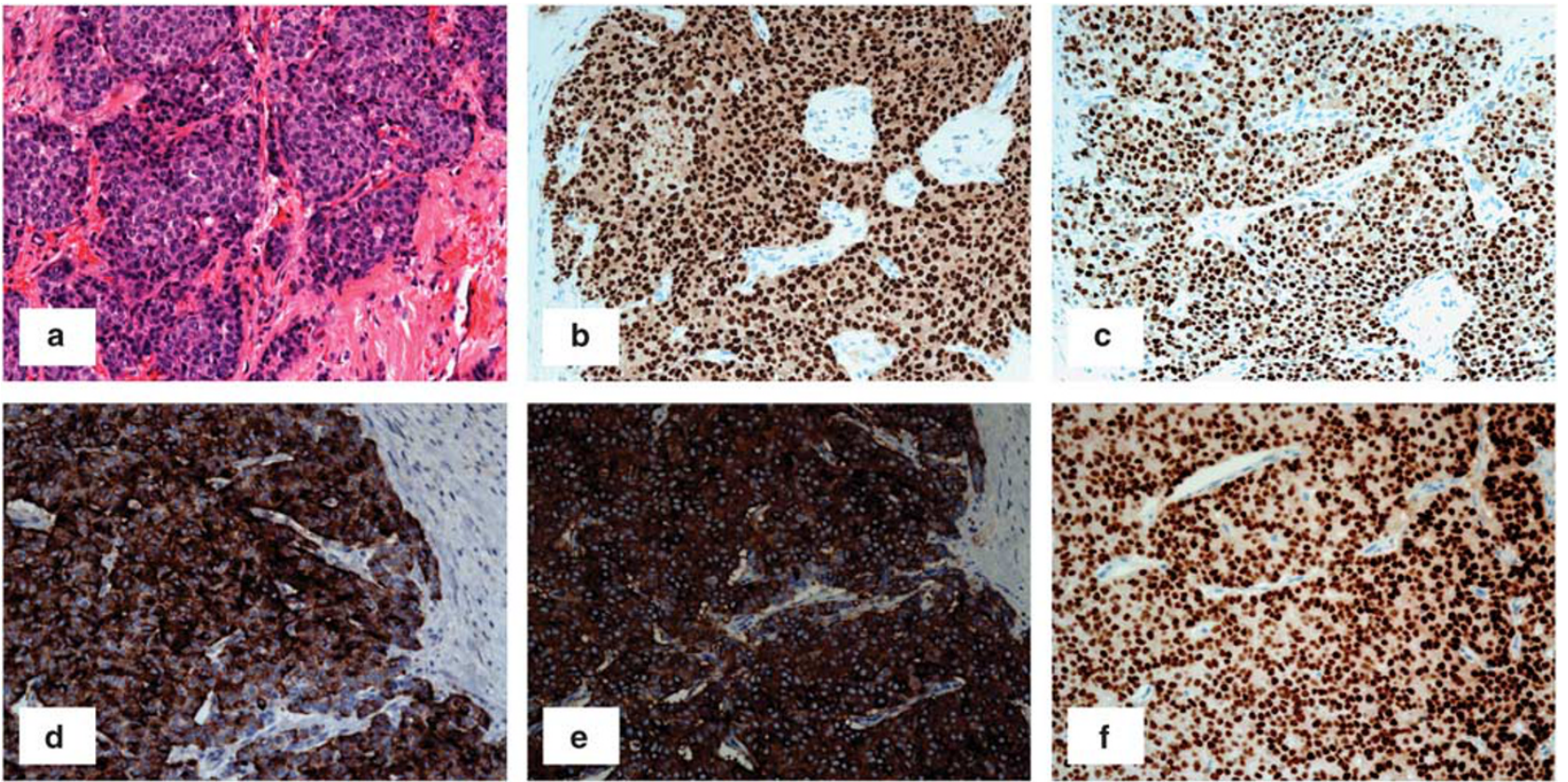

Figure 2 Invasive mammary carcinoma with neuroendocrine differentiation (magnification $\times 200$ ); (a) H\&E stain showing variably sized solid nests of tumor cells; (b) diffuse and strong immunoreactivity for ER; (c) diffuse and strong immunoreactivity for PR; (d) diffuse and strong immunoreactivity for synaptophysin; (e) diffuse and strong immunoreactivity for chromogranin; and (f) diffuse and strong immunoreactivity for GATA3.

surrounding breast tissue, whereas a significant number of invasive mammary carcinomas with neuroendocrine differentiation demonstrated associated in situ carcinoma. Hence, the presence of an in situ component favors the diagnosis of invasive mammary carcinoma with neuroendocrine differentiation, though the absence of in situ disease does not preclude the diagnosis of invasive mammary carcinoma with neuroendocrine differentiation. In evaluating the presence or absence of an in situ component, it should be noted that metastatic neuroendocrine neoplasms can exhibit a growth pattern that mimics in situ disease. ${ }^{9}$ In this situation, application of immunohistochemical markers for myoepithelial cells can aid in distinguishing true in situ carcinoma from invasive carcinoma mimicking in situ disease. In our study, we observed three such cases of metastatic neuroendocrine neoplasm where immunostains for myoepithelial markers were performed to exclude the presence of an in situ component. (3) All metastatic high-grade neuroendocrine carcinomas exhibited either smallcell or large-cell neuroendocrine carcinoma morphology. In contrast, invasive mammary carcinomas with neuroendocrine differentiation did not display typical features of small-cell carcinoma or large-cell neuroendocrine carcinoma. Accordingly, a tumor in the breast with classic small-cell or largecell neuroendocrine carcinoma features, in the absence of associated areas of usual invasive mammary carcinoma or in situ carcinoma, should raise the possibility of a metastatic high-grade neuroendocrine carcinoma, though primary small- cell carcinomas and large-cell neuroendocrine carcinomas in the breast do exist, albeit rarely. ${ }^{25,31-34}$

It is possible that a metastatic high-grade neuroendocrine carcinoma to the breast might be misinterpreted as a basaloid breast carcinoma (with or without neuroendocrine differentiation). Our control group of invasive mammary carcinomas with neuroendocrine differentiation did not include any tumors with a predicted basaloid molecular phenotype. (One tumor was triple-negative, but it lacked immunoreactivity for either cytokeratin $5 / 6$ or epidermal growth factor receptor, considered to be markers for true basaloid tumors.) Our selection criteria for the control group (requiring morphologic features suggestive of neuroendocrine differentiation and positive staining for at least one marker of neuroendocrine differentiation in more than $50 \%$ of the tumor cells) may have excluded cases of basaloid breast carcinoma with neuroendocrine differentiation.

Immunohistochemistry is helpful in distinguishing metastatic neuroendocrine neoplasms to the breast from primary mammary carcinomas, including in situ carcinomas, invasive mammary carcinomas, and invasive mammary carcinomas with neuroendocrine differentiation. ${ }^{35-38}$ In contrast to usual invasive mammary carcinomas (without neuroendocrine differentiation), metastatic neuroendocrine neoplasms (with the exception of some metastatic high-grade neuroendocrine carcinomas) are strongly and diffusely positive for neuroendocrine markers (synaptophysin and chromogranin). As discussed previously, immunostains for 
myoepithelial markers (such as p63 and smooth muscle myosin) can be utilized to differentiate metastatic neuroendocrine neoplasms from in situ carcinoma.

Neuroendocrine markers, however, are not helpful when the histologic differential diagnosis is between invasive mammary carcinoma with neuroendocrine differentiation and metastatic neuroendocrine neoplasm, since both may exhibit diffuse, strong positivity for neuroendocrine markers. In this setting, a panel of site-specific lineage markers (TTF-1 for pulmonary origin, CDX2 for gastrointestinal tract origin, PAX8/PAX6 for gastropancreatic and duodenal origin, and ER/PR, mammaglobin, GCDFP-15 and GATA3 for mammary origin) may be helpful in distinguishing metastatic neuroendocrine neoplasms (particularly well-differentiated neuroendocrine tumors) from invasive mammary carcinomas with neuroendocrine differentiation. ${ }^{35-52}$ In this study, CDX2 was positive in all examined metastatic neuroendocrine neoplasms of small bowel origin, TTF-1 was positive in $70 \%$ of examined metastatic neuroendocrine neoplasms of lung origin, and ER and GATA3 were positive in 91 and $100 \%$ of invasive mammary carcinomas with neuroendocrine differentiation, respectively. Similarly, in the study by Perry et al. ${ }^{9}$ (which with the exception of one high-grade neuroendocrine carcinoma consisted entirely of well-differentiated neuroendocrine tumors), all metastatic neuroendocrine neoplasms from the gastrointestinal tract expressed CDX2 and $60 \%$ of tumors originating from the lung expressed TTF-1. In addition, these authors found that the majority $(82 \%)$ of metastatic neuroendocrine neoplasms were negative for cytokeratin 7 , and all were negative for GCDFP-15 and mammaglobin. Immunostains for GCDFP-15 and/or mammaglobin were performed in only two of our metastatic neuroendocrine neoplasm cases, with negative results. However, we found that all of the nine metastatic neuroendocrine neoplasms stained for GATA3 were negative for this immunohistochemical stain, which is a more sensitive and specific marker for carcinomas of mammary origin than GCDFP-15 or mammaglobin. GATA3 expression is well recognized in breast carcinomas and urothelial carcinomas and more recently has also been described in a variety of other tumors, including (but not limited to) certain renal epithelial tumors, cutaneous squamous cell carcinomas, certain skin adnexal and salivary gland tumors, mesotheliomas, and autonomic nervous system tumors such as paragangliomas, pheochromocytomas, and neuroblastic tumors (neuroblastomas, ganglioneuroblastomas, and ganglioneuromas), but its expression in extramammary epithelial neuroendocrine tumors has not been described. ${ }^{4,50-52}$ Hence, GATA3 positivity in a breast tumor with neuroendocrine morphology would argue against a metastatic neuroendocrine neoplasm and in favor of a primary invasive mammary carcinoma with neuroendocrine differentiation.
While lineage-specific markers such as TTF-1, CDX2, and ER may be helpful in distinguishing metastatic neuroendocrine neoplasms (particularly well-differentiated neuroendocrine tumors) to the breast from invasive mammary carcinomas with neuroendocrine differentiation and in ascertaining the primary site of origin of a metastatic neuroendocrine neoplasm to the breast, the identification of the site of origin continues to be challenging due to considerable overlap in the immunohistochemical expression profiles of various neuroendocrine tumors. ${ }^{53}$ For example, 2 (13\%) of our 16 metastatic neuroendocrine neoplasms tested for ER expression were positive for ER. Likewise, Perry et al. ${ }^{9}$ observed ER positivity in 11\% of their cases of metastatic neuroendocrine neoplasm to the breast. Other investigators have also reported ER and PR positivity in small intestinal, pancreatic, pulmonary, and ovarian neuroendocrine tumors. ${ }^{38-40}$ Similarly, CDX2, a marker suggestive of intestinal origin, may be positive in pancreatic and ovarian neuroendocrine tumors. ${ }^{43}$ For high-grade neuroendocrine carcinomas, immunohistochemistry is usually not reliable in determining the primary site of origin. For example, TTF-1 is positive in $80 \%$ of extrapulmonary small-cell carcinomas. ${ }^{39}$ Likewise, focal weak to diffuse and strong immunoexpression of ER and PR can be noted in endometrial and cervical high-grade neuroendocrine carcinomas. ${ }^{44-46}$ Therefore, in the absence of a relevant clinical history, determining whether an invasive carcinoma with neuroendocrine differentiation in the breast is primary or metastatic and suggesting the site of origin of a metastatic neuroendocrine neoplasm in the breast based solely on the results of immunohistochemical studies can be problematic.

In conclusion, the histopathologic and immunohistochemical distinction of metastatic neuroendocrine neoplasm to the breast from invasive mammary carcinoma with neuroendocrine differentiation can be challenging. If a neoplasm in the breast exhibits neuroendocrine cytoarchitectural features, with diffuse, strong staining for neuroendocrine marker (s), absence of associated in situ carcinoma, and negativity for ER and GATA3, the possibility of a metastatic neuroendocrine neoplasm should be strongly entertained, warranting a meticulous search for a primary extramammary neuroendocrine neoplasm, if not already known. Primary breast carcinomas with classic small-cell or large-cell neuroendocrine morphologic features are rare; the finding of a tumor in the breast with such features should therefore raise the possibility of a metastatic high-grade neuroendocrine carcinoma, necessitating correlation with the clinical history and imaging findings. Recognition of metastatic neuroendocrine tumors to the breast is critical for the selection of appropriate clinical treatment for these patients. 


\section{Acknowledgments}

The authors acknowledge Ira Bleiweiss, MD and Farnaz Dadmanesh, MD for contributing cases.

\section{Disclosure/conflict of interest}

The authors declare no conflict of interest.

\section{References}

1 Georgiannos SN, Chin J, Goode AW et al. Secondary neoplasms of the breast: a survey of the $20^{\text {th }}$ century. Cancer 2001;92:2259-2266.

2 Hajdu SI, Urban JA. Cancers metastatic to the breast. Cancer 1972;29:1691-1696.

3 Chaignaud B, Hall TJ, Powers C et al. Diagnosis and natural history of extramammary tumors metastatic to the breast. J Am Coll Surg 1994;179:49-53.

4 DeLair D, Corben AD, Catalano JP et al. Non-mammary metastases to the breast and axilla: a study of 85 cases. Mod Pathol 2013;26:343-349.

5 Lee AH. The histological diagnosis of metastases to the breast from extramammary malignancies. J Clin Pathol 2007;60:1333-1341.

6 Shetty MR. Carcinoid tumour of the breast. Eur J Surg Oncol 1996;22:307.

7 Kashlan RB, Powell RW, Nolting SF. Carcinoid and other tumors metastatic to the breast. J Surg Oncol 1982;20:25-30.

8 Shetty MR, Ahmed MI. 12 cases of carcinoid tumors metastatic to the breast have been reported. Gynecol Oncol 1995;57:436-437.

9 Perry KD, Reynolds C, Rosen DG et al. Metastatic neuroendocrine tumour in the breast: a potential mimic of in-situ and invasive mammary carcinoma. Histopathology 2011;59:619-630.

10 Chodoff RJ. Solitary breast metastasis from a carcinoid of the ileum. Am J Surg 1965;309:814-815.

11 Hawley PR. A case of secondary carcinoid tumors in both breasts following excision of primary carcinoid tumour of the duodenum. Br J Surg 1966;53:818-820.

12 Adams RF, Parulekar V, Hughes C et al. Radiologic characteristics and management of screen-detected metastatic carcinoid tumor of the breast: a case report. Clin Breast Cancer 2009;9:189-192.

13 Ahlman H, Larsson I, Gronstad K et al. A case of midgut carcinoid with breast metastasis and cellular localization of serotonin and substance P. J Surg Oncol 1986;31:170-173.

14 Di Palma S, Andreola S, Lombardi L et al. Ileal carcinoid metastatic to the breast. Report of a case. Tumori 1988;74:321-327.

15 Fishman A, Kim H, Girtanner RE et al. Solitary breast metastasis as first manifestation of ovarian carcinoid tumor. Gynecol Oncol 1994;54:222-226.

16 Geyer HL, Viney J, Karlin N. Metastatic carcinoid presenting as a breast lesion. Curr Oncol 2010;17: $73-77$.

17 Harrist TJ, Kalisher L. Breast metastasis: an unusual manifestation of a malignant carcinoid tumor. Cancer 1977;40:3102-3106.

18 Mosunjac MB, Kochhar R, Mosunjac MI et al. Primary small bowel carcinoid tumor with bilateral breast metastases: report of 2 cases with different clinical presentations. Arch Pathol Lab Med 2004;128: 292-297.

19 Ordonez NG, Manning JT Jr, Raymond AK. Argentaffin endocrine carcinoma (carcinoid) of the pancreas with concomitant breast metastasis: an immunohistochemical and electron microscopic study. Hum Pathol 1985;16:746-751.

20 Rubio IT, Korourian S, Brown H et al. Carcinoid tumor metastatic to the breast. Arch Surg 1998;133: 1117-1119.

21 Schurch W, Lamoureux E, Lefebvre R et al. Solitary breast metastasis: first manifestation of an occult carcinoid of the ileum. Virchows Arch A Pathol Anat Histol 1980;386:117-124.

22 Turner M, Gallager HS. Occult appendiceal carcinoid. Report of a case with fatal metastases. Arch Pathol 1969;88:188-190.

23 Upalakalin JN, Collins LC, Tawa N et al. Carcinoid tumors in the breast. Am J Surg 2006;191:799-805.

24 Warner TF, Seo IS. Bronchial carcinoid appearing as a breast mass. Arch Pathol Lab Med 1980;104: 531-534.

25 Ellis IO, Schnitt SJ, Sastre-Garau X et al. Invasive breast carcinoma. In: Tavassoli FA, Devilee P (eds). Pathology and Genetics. Tumours of the Breast and Female Genital Organs. IARC Press: Lyon, France, 2003, pp 32-34.

26 Rindi G, Arnold R, Bosman FT et al. Nomenclature and classification of neuroendocrine neoplasms of the digestive system. In: Bosman FT, Carneiro F, Hruban $\mathrm{RH}$ et al. (eds). WHO Classification of Tumours of the Digestive System. IARC Press: Lyon, France, 2010, p 13.

27 Travis WD, Brambilla E, Muller-Hermelink HK et al. (eds). WHO Classification of Tumors. Pathology and Genetics of Tumours of the Lung, Pleura, Thymus and Heart. IARC Press: Lyon, France, 2004.

28 Edge SB, Byrd DR, Compton CC et al. (eds). AJCC Cancer Staging Manual, 7th edn. Springer: Chicago, IL, 2009, pp 184.

29 Dowsett M, Nielsen TO, A'Hern R et al. Assessment of Ki-67 in breast cancer: recommendations from the International Ki67 in Breast Cancer working group. J Natl Cancer Inst 2011;103:1656-1664.

30 Dhall D, Mertens R, Bresee C et al. Ki-67 proliferative index predicts progression-free survival of patients with well-differentiated ileal neuroendocrine tumors. Hum Pathol 2012;43:489-495.

31 Wei B, Ding T, Xing Y et al. Invasive neuroendocrine carcinoma of the breast: a distinctive subtype of aggressive mammary carcinoma. Cancer 2010;116: 4463-4473.

32 Shin SJ, DeLellis RA, Ying L et al. Small cell carcinoma of the breast. A clinicopathologic and immunohistochemical study of nine patients. Am J Surg Pathol 2000;24:1231-1238.

33 Miremadi A, Pinder SE, Lee AH et al. Neuroendocrine differentiation and prognosis in breast adenocarcinoma. Histopathology 2002;40:215-222.

34 Bussolati G, Badve S. Carcinomas with neuroendocrine features. In: Lakhani SR, Ellis IO, Schnitt SJ et al. (eds). WHO Classification of Tumours of the Breast. IARC Press: Lyon, France, 2012, pp 62-63.

35 Keshgegian AA, Wheeler JE. Estrogen receptor protein in malignant carcinoid tumor: a report of 2 cases. Cancer 1980;45:293-296. 
36 Sica G, Wagner PL, Altorki N et al. Immunohistochemical expression of estrogen and progesterone receptors in primary pulmonary neuroendocrine tumors. Arch Pathol Lab Med 2008;132:1889-1895.

37 Arnason T, Sapp HL, Barnes PJ et al. Immunohistochemical expression and prognostic value of ER, PR and HER2/neu in pancreatic and small intestinal neuroendocrine tumors. Neuroendocrinology 2011;93: 249-258.

38 Srivastava A, Hornick JL. Immunohistochemical staining for CDX-2, PDX-1, NESP-55, and TTF-1 can help distinguish gastrointestinal carcinoid tumors from pancreatic endocrine and pulmonary carcinoid tumors. Am J Surg Pathol 2009;33:626-632.

39 Kaufmann O, Dietel M. Expression of thyroid transcription factor-1 in pulmonary and extrapulmonary small cell carcinomas and other neuroendocrine carcinomas of various primary sites. Histopathology 2000;36: $415-420$

40 Hayashi T, Haba R, Kushida Y et al. Cytopathologic characteristics of the primary strumal carcinoid tumor of the ovary: a case report with emphasis on differential diagnostic considerations. Diagn Cytopathol 2013;41: 812-816.

41 Hamazaki S, Okino T, Tsukayama C et al. Expression of thyroid transcription factor-1 in strumal carcinoid and struma ovarii: an immunohistochemical study. Pathol Int 2002;52:458-462.

42 Cai YC, Banner B, Glickman J et al. Cytokeratin 7 and 20 and thyroid transcription factor 1 can help distinguish pulmonary from gastrointestinal carcinoid and pancreatic endocrine tumors. Hum Pathol 2001;32: 1087-1093.

43 Rabban JT, Lerwill MF, McCluggage WG et al. Primary ovarian carcinoid tumors may express CDX-2: a potential pitfall in distinction from metastatic intestinal carcinoid tumors involving the ovary. Int J Gynecol Pathol 2009;28:41-48.

44 Tangjitgamol S, Ramirez PT, Sun CC et al. Expression of HER-2/neu, epidermal growth factor receptor, vascular endothelial growth factor, cyclooxygenase-2, estrogen receptor, and progesterone receptor in small cell and large cell neuroendocrine carcinoma of the uterine cervix: a clinicopathologic and prognostic study. Int J Gynecol Cancer 2005;15: 646-656.

45 Terada T. Large cell neuroendocrine carcinoma with sarcomatous changes of the endometrium: a case report with immunohistochemical studies and molecular genetic study of KIT and PDGFRA. Pathol Res Pract 2010;206:420-425.

46 Zhao C, Bratthauer GL, Barner R et al. Comparative analysis of alternative and traditional immunohistochemical markers for the distinction of ovarian sertoli cell tumor from endometrioid tumors and carcinoid tumor: A study of 160 cases. Am J Surg Pathol 2007;31: $255-266$.

47 Kouros-Mehr Hosein, Slorach EM, Sternlicht MD et al. GATA-3 maintains the differentiation of the luminal cell fate in the mammary gland. Cell 2006;127: 1041-1055.

48 Koo J, Mertens RB, Mirocha JM et al. Value of Islet 1 and PAX8 in identifying metastatic neuroendocrine tumors of pancreatic origin. Mod Pathol. 2012;25: 893-901.

49 Lai JP, Mertens RB, Mirocha J et al. Comparison of PAX6 and PAX8 as immunohistochemical markers for pancreatic neuroendocrine tumors. Endocr Pathol. 2015;26:54-62.

50 Miettinen M, McCue PA, Sarlomo-Rikala M et al. GATA3: a multispecific but potentially useful marker in surgical pathology: a systematic analysis of 2500 epithelial and nonepithelial tumors. Am J Surg Pathol 2014;38:13-22.

51 Nonaka D, Wang BY, Edmondson D et al. A study of GATA3 and phox2b expression in tumors of the autonomic nervous system. Am J Surg Pathol 2013;37: 1236-1241.

52 So JS, Epstein JI. GATA3 expression in paragangliomas: a pitfall potentially leading to misdiagnosis of urothelial carcinoma. Mod Pathol 2013;26:1365-1370.

53 Czeczok TW, Gailey MP, Hornick JL et al. High-grade neuroendocrine carcinomas are characterized by marked transcription factor lineage infidelity: An evaluation of 36 diagnostic markers in 83 tumors. Mod Pathol 2014;27:152A. 